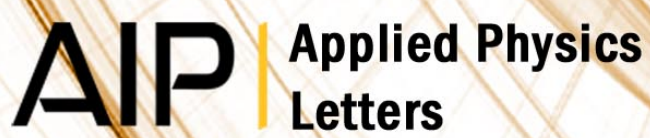

\section{Room-temperature organic-based spin polarizer}

Bin Li, Chi-Yueh Kao, Yu Lu, Jung-Woo Yoo, Vladimir N. Prigodin et al.

Citation: Appl. Phys. Lett. 99, 153503 (2011); doi: 10.1063/1.3651329

View online: http://dx.doi.org/10.1063/1.3651329

View Table of Contents: http://apl.aip.org/resource/1/APPLAB/v99/i15

Published by the AIP Publishing LLC.

\section{Additional information on Appl. Phys. Lett.}

Journal Homepage: http://apl.aip.org/

Journal Information: http://apl.aip.org/about/about_the_journal

Top downloads: http://apl.aip.org/features/most_downloaded

Information for Authors: http://apl.aip.org/authors

\section{ADVERTISEMENT}
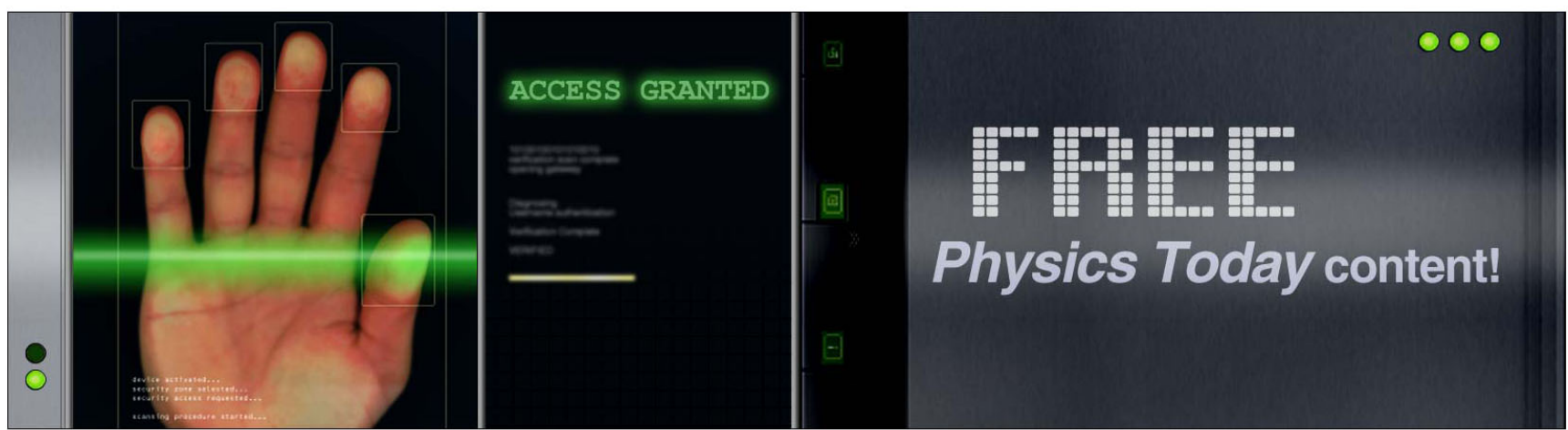


\title{
Room-temperature organic-based spin polarizer
}

\author{
Bin Li, ${ }^{1}$ Chi-Yueh Kao, ${ }^{2}$ Yu Lu, ${ }^{2}$ Jung-Woo Yoo, ${ }^{3}$ Vladimir N. Prigodin,,${ }^{1,4}$ \\ and Arthur J. Epstein ${ }^{1,2, a)}$ \\ ${ }^{1}$ Department of Physics, The Ohio State University, Columbus, Ohio 43210-1117, USA \\ ${ }^{2}$ Department of Chemistry, The Ohio State University, Columbus, Ohio 43210-1173, USA \\ ${ }^{3}$ Department of Mechanical and Advanced Materials Engineering, UNIST, Ulsan 689-805, Korea \\ ${ }^{4}$ Ioffe Institute, 194021 St. Petersburg, Russia
}

(Received 1 June 2011; accepted 23 September 2011; published online 11 October 2011)

\begin{abstract}
We report a magnetic tunnel junction operating at room-temperature with organic magnetic semiconductor $\mathrm{V}[\mathrm{TCNE}]_{x}(\mathrm{x} \sim 2$, TCNE: tetracyanoethylene $)$ and $\mathrm{Fe}$ as the spin polarizer and analyzer while $10 \mathrm{~nm}$ rubrene layer serves as the tunnel barrier between them. At room-temperature, the magnetoresistance (MR) presents $16.7 \%$ of its peak value at $100 \mathrm{~K}$. We observed sign inversion of MR with increasing temperature, while the sign of the MR is independent of the polarity of the bias voltages. Our results suggest that $\mathrm{V}[\mathrm{TCNE}]_{x}$ is a promising material for room-temperature spintronic applications. (C) 2011 American Institute of Physics. [doi:10.1063/1.3651329]
\end{abstract}

Recently, spin injection and detection in carbon-based materials have been a focus of attention, motivated by the long spin-relaxation time in these materials. ${ }^{1,2}$ Spin valves which consist of two magnetic layers with distinct coercivities decoupled by a spacer have often been used to demonstrate spin-polarized transport. Organic semiconductors have been employed as the spin transport layers or tunneling barriers, such as tris(8-hydroxyquinoline)-aluminum $\left(\mathrm{Alq}_{3}\right)$ (Refs. 3-5) and rubrene $\left(\mathrm{C}_{42} \mathrm{H}_{28}\right){ }^{6-8}$ However, there is a fundamental problem so-called "conductivity mismatch" for spin injection from ferromagnetic metals into semiconductors. ${ }^{9}$ The development of organic-based magnets is promising to provide a pathway to circumvent this problem. ${ }^{10}$ The combination of organic magnets and organic semiconductors will also be likely to give rise the next-generation lightweight, mechanically flexible and low-cost spintronic devices.

$\mathrm{V}[\mathrm{TCNE}]_{x}(\mathrm{x} \sim 2, \mathrm{TCNE}:$ tetracyanoethylene $)$ with magnetic ordering temperature $T_{c} \sim 400 \mathrm{~K}$ is the first reported room-temperature organic-based magnet. ${ }^{11}$ The antiferromagnetic coupling between the $\mathrm{V}^{2+}$ ion's three unpaired electrons in $3 d\left(t_{2 g}\right)$ orbitals and the $[\mathrm{TCNE}]^{-}$ anion's unpaired electron in $\pi^{*}$ orbital gives rise to a net spin of $S=1 / 2$ for the repeat unit. ${ }^{12}$ The chemical structure of TCNE is shown in Figure 1(b). This material can be grown as a thin film by chemical vapor deposition (CVD) or molecular layer deposition (MLD). ${ }^{13,14}$ Optical detection of spin injection from $\mathrm{V}[\mathrm{TCNE}]_{x}$ into a GaAs/AlGaAs lightemitting diode has been realized. ${ }^{15}$ We have reported spin injection and detection using $\mathrm{V}[\mathrm{TCNE}]_{x}$ both in a hybrid tunnel junction and an all-organic-based tunnel junction. ${ }^{16,17}$ However, the operation of the devices has been limited at low temperature, which is an obstacle for the development of practical applications. In this letter, we report experimental results on a $\mathrm{V}[\mathrm{TCNE}]_{x}$-based spin valve operating up to room-temperature.

A spin valve is a sandwich device which consists of two ferromagnetic (FM) contacts with different coercivities sepa-

${ }^{a)}$ Electronic mail: epstein@physics.osu.edu. rated by a nonmagnetic spacer. The device can switch between parallel and antiparallel magnetization configurations by sweeping an external magnetic field. In our experiments, $\mathrm{V}[\mathrm{TCNE}]_{x}$ and $\mathrm{Fe}$ were chosen as the two magnetic contacts of the spin valve, while rubrene acted as the tunnel barrier, as illustrated in Figure 1(a). We fabricated the spin valve by thermal evaporation using in situ shadow masks. To begin the device fabrication, glass substrates were cleaned ultrasonically and dried with nitrogen. Then organic solvent residue was removed by a UV-ozone cleaner. All the cleaning procedures were performed in a Class 1000 cleanroom. Right after the cleaning, the substrates were transferred into a vacuum chamber integrated in an argon glovebox for film depositions. The base pressure of the chamber was $5 \times 10^{-7}$ Torr. $50 \mathrm{~nm}$ of Fe was deposited by e-beam evaporation at a rate of $0.2 \AA / \mathrm{s}$. $10 \mathrm{~nm}$ of rubrene (chemical structure shown in Figure 1(c)) was deposited thermally using a Knudsen cell at a controlled temperature $\left(\sim 105^{\circ} \mathrm{C}\right)$. We have reported that a single $5 \mathrm{~nm}$ rubrene layer was too thin to

(a)

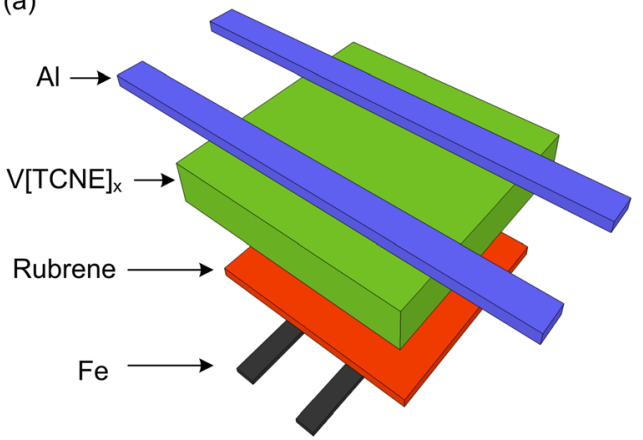

(b)

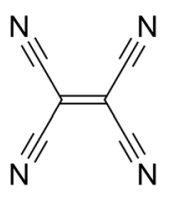

(c)

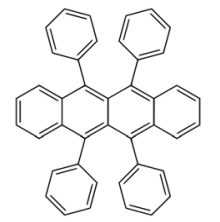

FIG. 1. (Color online) (a) Schematic view of the device structure. (b) Chemical structure of TCNE (tetracyanoethylene). (c) Chemical structure of rubrene $\left(\mathrm{C}_{42} \mathrm{H}_{28}\right)$. 


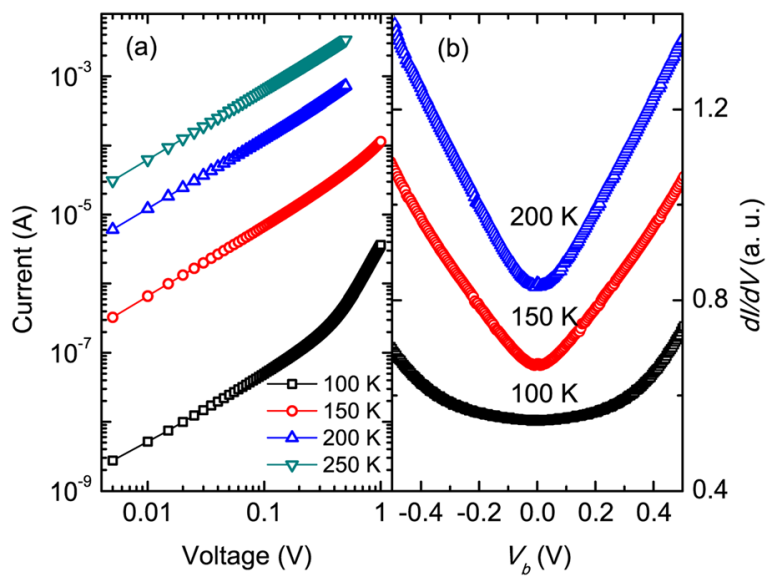

FIG. 2. (Color online) (a) $I-V$ characteristics of the spin valve with the structure of $\mathrm{Fe}(50 \mathrm{~nm}) /$ rubrene $(10 \mathrm{~nm}) / \mathrm{V}[\mathrm{TCNE}]_{x}(300 \mathrm{~nm})$ at different temperatures: $100 \mathrm{~K}, 150 \mathrm{~K}, 200 \mathrm{~K}$, and $250 \mathrm{~K}$. (b) $d I / d V$ as a function of bias voltage $V_{b}$ at different temperatures: $100 \mathrm{~K}, 150 \mathrm{~K}$, and $200 \mathrm{~K}$.

provide a uniform coverage or to form a good barrier. ${ }^{16}$ Lin et al. also reported that maximum tunneling thickness for rubrene is about $15 \mathrm{~nm}^{18}$ The V[TCNE $]_{x}$ layer was grown by low temperature $\left(\sim 40^{\circ} \mathrm{C}\right) \mathrm{CVD}$ with a thickness of about $300 \mathrm{~nm}$. Another $30 \mathrm{~nm}$ of $\mathrm{Al}$ was deposited as the top contact. The effective junction area was $1 \mathrm{~mm} \times 1 \mathrm{~mm}$. The electric and magnetic measurements were performed in a physical property measurement system (PPMS) from Quantum Design with Keithley 2400 sourcemeter. The magnetic hysteresis loops were measured with a superconducting quantum interference device (SQUID).

Figure 2(a) shows the typical current-voltage (I-V) characteristics of the device at different temperatures, which are similar with the reported LSMO/LAO/rubrene/Fe magnetic tunnel junction. ${ }^{7}$ At low temperature and low bias voltage, the device current is dominated by tunneling through defect states in the energy gap. ${ }^{7}$ The carriers in these defect states could enter HOMO/LUMO levels via strong electric field as we increase the bias voltage, leading to the increase of the device current. At higher temperature, phonon interaction, such as phonon-assisted field emission, ${ }^{7}$ starts to play a role, giving rise to the temperature dependence. The absence of

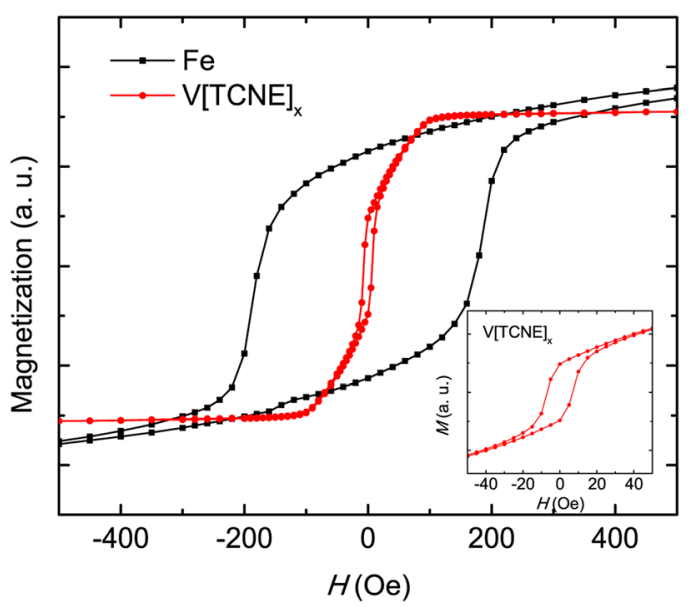

FIG. 3. (Color online) Coercive fields of Fe $(50 \mathrm{~nm})$ film and V[TCNE $]_{x}$ $(300 \mathrm{~nm})$ film measured separately by SQUID at $100 \mathrm{~K}$. The inset figure shows enlarged detail of the hysteresis loop for $\mathrm{V}[\mathrm{TCNE}]_{x}$ film.

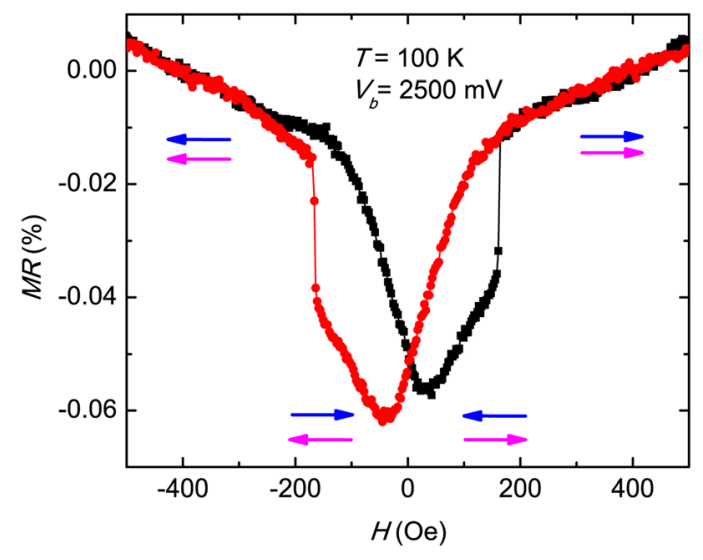

FIG. 4. (Color online) MR measurements of the spin valve with the structure of $\mathrm{Fe}(50 \mathrm{~nm}) /$ rubrene $(10 \mathrm{~nm}) / \mathrm{V}[\mathrm{TCNE}]_{x}(300 \mathrm{~nm})$ at $100 \mathrm{~K}$ with a bias of $2500 \mathrm{mV}$.

zero bias anomalies in the conductance vs bias plot $(d I / d V$ vs $V$, see Figure 2(b)) suggests that the rubrene formed a good tunnel barrier, as discussed in literatures. ${ }^{4,6}$ Figure 3 shows the hysteresis loops of the two FM layers measured by SQUID separately. At $100 \mathrm{~K}, 50 \mathrm{~nm}$ Fe film has a coercive field of $160 \mathrm{Oe}$ and $\mathrm{V}[\mathrm{TCNE}]_{x}$ 's coercive field is about $5 \mathrm{Oe}$. The resistance of a spin valve varies depending on the relative alignment of the magnetization of the two FM layers. The magnetoresistance (MR) value is defined as $M R=\left(R_{A P}\right.$ $\left.-R_{P}\right) / R_{P}$, where $R_{A P}$ and $R_{P}$ are the device resistance corresponding to antiparallel (AP) and parallel (P) configurations, respectively. The typical MR curves obtained from our devices are shown in Figure 4. At high applied magnetic field, the two FM layers align parallel with the external field. When the field reaches the coercive field of one of the FM layer, the device resistance has a sharp change as the two FM align antiparallel. The device showed negative MR at $100 \mathrm{~K}$, which corresponds to lower device resistance for antiparallel alignment. The sign of the MR is independent of bias polarity. At $200 \mathrm{~K}$, the MR is still clearly negative, as shown in Figure 5(a). Starting from $250 \mathrm{~K}$, we can see a positive part contributing to the total MR. We have observed this
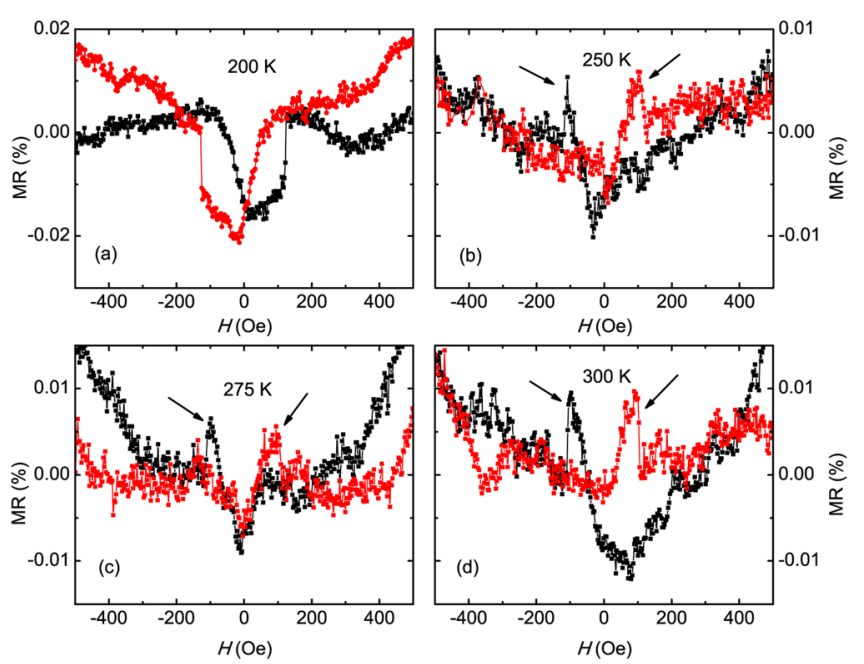

FIG. 5. (Color online) MR measurements at $500 \mathrm{mV}$ bias voltage at different temperature: (a) $200 \mathrm{~K}$, (b) $250 \mathrm{~K}$, (c) $275 \mathrm{~K}$, and (d) $300 \mathrm{~K}$. The arrows highlight the MR peaks. 


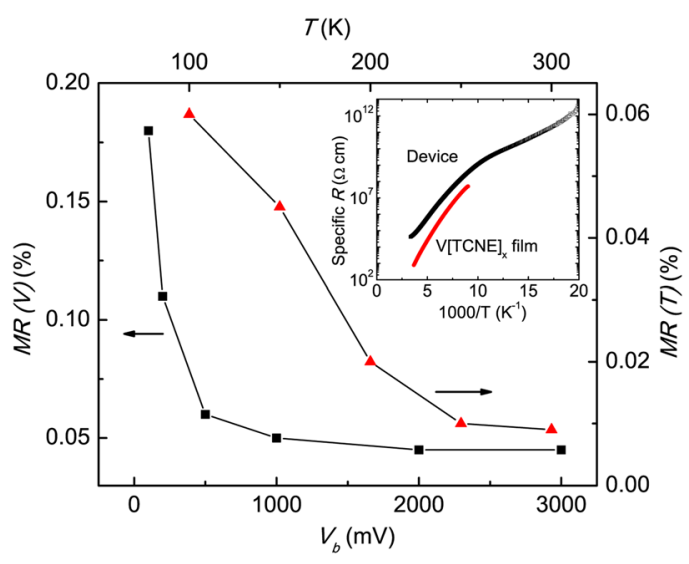

FIG. 6. (Color online) (Black square) Bias dependence of MR values at $100 \mathrm{~K}$. (Red triangle) Temperature dependence of MR values at $500 \mathrm{mV}$. The inset shows temperature dependent specific resistance $(\Omega \cdot \mathrm{cm})$ of the spin valve device and $\mathrm{V}[\mathrm{TCNE}]_{x}$ film measured separately.

feature at temperature range from $250 \mathrm{~K}$ to $300 \mathrm{~K}$ (see Figures $5(\mathrm{~b})-5(\mathrm{~d})$ ). The temperature dependence of MR value is presented in Figure 6, obtained by measuring MR under $500 \mathrm{mV}$ at different temperatures, showing the maximum MR $0.06 \%$ occurs at $100 \mathrm{~K}$. The device resistance increased as we lowered the temperature (see the inset of Figure 6), indicating the tunnel barrier is free of pinholes. ${ }^{19}$ Below $100 \mathrm{~K}$, the device resistance became too high for us to detect any MR. The decrease of MR with increasing temperature is a common behavior for magnetic tunnel junction and can be explained by the increase of defect density and inelastic transport at high temperature. ${ }^{6}$ Our device kept showing spin valve effect up to $300 \mathrm{~K}$, with a MR value of $0.01 \%$. Although the absolute MR value at room-temperature is small, it remains $16.7 \%$ of its peak value obtained at $100 \mathrm{~K}$. The bias dependence of the MR values at $100 \mathrm{~K}$ is also presented in Figure 6. At $100 \mathrm{~K}$, the maximum MR obtained is $0.18 \%$ under $100 \mathrm{mV}$. The rapid decrease of MR value with increasing bias is observed in various magnetic tunnel junctions. 6 ,20

As a control experiment, we measured the resistance of the $50 \mathrm{~nm}$ Fe film as a function of applied magnetic field separately using the exact same measurement set-up as the spin valve. We did not observe any MR from the Fe film. It has been reported that the MR of CVD-grown V[TCNE $]_{x}$ film increases linearly with increasing external magnetic field with no shift. ${ }^{12,21}$ The estimated MR of V[TCNE $]_{x}$ film at 100 Oe would be two order of magnitude smaller than the MR we observed. Thus, we believe the MR in our measurements is from the spin-dependent tunneling. We want to emphasize here that the interface plays a crucial role in the tunneling, as pointed by Barraud et al..$^{5}$ In our previous reported LSMO/rubrene/V[TCNE $]_{x}$ tunnel junction, we have shown that a single $5 \mathrm{~nm}$ rubrene could not provide a good tunnel barrier while hybrid barrier $\mathrm{LAO} /$ rubrene give rise to a clear MR signal. ${ }^{16}$ Here we suggest that the reason accounting for the relatively small MR values may be the imperfect interface, as it was pointed by a number of authors. ${ }^{5,22}$ However, due to the sensitivity of the V[TCNE $]_{x}$ film, the interface study between the V $[\mathrm{TCNE}]_{x}$ and the rubrene is not easily accessible at this moment. New methods need to be developed to directly probe the interface between the ferromagnetic contact and the organic spacer. In our experiments, we observed the sign inversion of the MR as we varied the temperature, and the sign of MR was independent of polarity of bias voltage. Previously Vinzelberg et $a l$. reported a MR sign inversion in dependence on the current in a $\mathrm{LSMO} / \mathrm{Alq}_{3} / \mathrm{Co}$ device. ${ }^{23}$ The reason for the sign inversion is not clear yet.

In summary, we demonstrated a spin valve working at room-temperature using organic magnetic semiconductor $\mathrm{V}[\mathrm{TCNE}]_{x}$ as the spin injector/detector. This result suggests that $\mathrm{V}[\mathrm{TCNE}]_{x}$ could be one of the promising candidates for next-generation room-temperature spintronic applications. Future studies on the interface would provide more room for improvement of the device performance.

B. L. thanks Dr. Chia-Yi Chen and Megan M. Harberts for their help with the CVD system. This work was supported in part by AFOSR Grant No. FA-9550-06-1-0175, DOE Grant Nos. DE-FG02-01ER45931, DE-FG0286ER45271, NSF Grant No. DMR-0805220, the Center for Emergent Materials (an NSF-MRSEC; Award Number DMR-0820414) at The Ohio State University and the Institute for Materials Research at The Ohio State University.

${ }^{1}$ V. A. Dediu, L. E. Hueso, I. Bergenti, and C. Taliani, Nature Mater. 8, 707 (2009).

${ }^{2}$ G. Szulczewski, S. Sanvito, and M. Coey, Nature Mater. 8, 693 (2009).

${ }^{3}$ Z. H. Xiong, D. Wu, Z. Valy Vardeny, and J. Shi, Nature 427, 821 (2004).

${ }^{4}$ T. S. Santos, J. S. Lee, P. Migdal, I. C. Lekshmi, B. Satpati, and J. S. Moodera, Phys. Rev. Lett. 98, 016601 (2007).

${ }^{5}$ C. Barraud, P. Seneor, R. Mattana, S. Fusil, K. Bouzehouane, C. Deranlot, P. Graziosi, L. Hueso, I. Bergenti, V. Dediu, F. Petroff, and A. Fert, Nature Phys. 6, 615 (2010).

${ }^{6}$ J. H. Shim, K. V. Raman, Y. J. Park, T. S. Santos, G. X. Miao, B. Satpati, and J. S. Moodera, Phys. Rev. Lett. 100, 226603 (2008).

${ }^{7}$ J.-W. Yoo, H. W. Jang, V. N. Prigodin, C. Kao, C. B. Eom, and A. J. Epstein, Phys. Rev. B 80, 205207 (2009).

${ }^{8}$ B. Li, J.-W. Yoo, C.-Y. Kao, H. W. Jang, C.-B. Eom, and A. J. Epstein, Org. Electron. 11, 1149 (2010).

${ }^{9}$ G. Schmidt, D. Ferrand, L. W. Molenkamp, A. T. Filip, and B. J. van Wees, Phys. Rev. B 62, R4790 (2000).

${ }^{10}$ J. S. Miller and A. J. Epstein, Angew. Chem. Int. Ed. Engl. 33, 385 (1994).

${ }^{11}$ J. M. Manriquez, G. T. Yee, R. S. McLean, A. J. Epstein, and J. S. Miller, Science 252, 1415 (1991).

${ }^{12}$ V. Prigodin, N. Raju, K. Pokhodnya, J. Miller, and A. Epstein, Adv. Mater. 14, 1230 (2002).

${ }^{13}$ K. I. Pokhodnya, A. J. Epstein, and J. S. Miller, Adv. Mater. 12, 410 (2000).

${ }^{14}$ C.-Y. Kao, J.-W. Yoo, and A. J. Epstein (unpublished).

${ }^{15}$ L. Fang, K. D. Bozdag, C.-Y. Chen, P. A. Truitt, A. J. Epstein, and E. Johnston-Halperin, Phys. Rev. Lett. 106, 156602 (2011).

${ }^{16}$ J.-W. Yoo, C.-Y. Chen, H. W. Jang, C. W. Bark, V. N. Prigodin, C. B. Eom, and A. J. Epstein, Nature Mater. 9, 638 (2010).

${ }^{17}$ B. Li, C.-Y. Kao, J.-W. Yoo, V. N. Prigodin, and A. J. Epstein, Adv. Mater. 23, 3382 (2011).

${ }^{18}$ R. Lin, F. Wang, J. Rybicki, M. Wohlgenannt, and K. A. Hutchinson, Phys. Rev. B 81, 195214 (2010).

${ }^{19}$ J. J. Åkerman, R. Escudero, C. Leighton, S. Kim, D. Rabson, R. W. Dave, J. Slaughter, and I. K. Schuller, J. Magn. Magn. Mater. 240, 86 (2002).

${ }^{20}$ J. S. Moodera and G. Mathon, J. Magn. Magn. Mater. 200, 248 (1999).

${ }^{21}$ N. P. Raju, T. Savrin, V. N. Prigodin, K. I. Pokhodnya, J. S. Miller, and A. J. Epstein, J. Appl. Phys. 93, 6799 (2003).

${ }^{22}$ J. S. Moodera, T. S. Santos, and K. V. Raman, Organic Spintronics, edited by Z. V. Vardeny (CRC Press, Boca Raton, 2010).

${ }^{23}$ H. Vinzelberg, J. Schumann, D. Elefant, R. B. Gangineni, J. Thomas, and B. Buchner, J. Appl. Phys. 103, 093720 (2008). 